\section{The diagnostic performance of renal \\ function-adjusted D-dimer testing in individuals suspected of having venous thromboembolism}

Renal impairment, a source of chronic hypercoagulability ${ }^{1}$ and inflammation, ${ }^{2}$ is known to reduce the specificity of the $\mathrm{D}$-dimer test in the diagnosis of venous thromboembolism (VTE) ${ }^{3}$ This leads to many false positives in such patients and consequently to additional costs, as well as the unnecessary exposure of patients with renal impairment to contrast dye used in computed tomographic pulmonary angiography. ${ }^{3}$

To address this issue, researchers have attempted to develop renal function-based thresholds for the $\mathrm{D}$-dimer test. ${ }^{4.6}$ The thresholds presented so far have varied greatly, which we surmised might have been due to unmeasured confounding factors. We therefore studied whether a renal function-adjusted D-dimer test could improve diagnostic performance for individuals with suspected VTE, and how the relationship between D-dimer levels and estimated glomerular filtration rates (eGFR) is affect- ed by age and C-reactive protein (CRP) levels. Both age and inflammation have previously been shown to affect D-dimer concentrations ${ }^{7}$ and renal function. ${ }^{8}$

Data from the VTEval project ${ }^{9}$ (NCTO2156401) - an investigator-initiated, observational, single-center, prospective cohort study of individuals with clinically suspected VTE - were analyzed. Patients $\geq 18$ years who were referred to the University Medical Center Mainz with suspected deep vein thrombosis or pulmonary embolism and who had an indication for imaging were enrolled and diagnosed using color Doppler ultrasonography or computed tomographic pulmonary angiography, respectively. All diagnoses of VTE were verified by board-certified senior angiologists or radiologists. The eGFR was calculated using the Chronic Kidney Disease Epidemiology Collaboration (CKD-EPI) 2009 equation. ${ }^{10}$ Diagnostic test characteristics [area under the receiver operating characteristic curve (AUC), sensitivity, specificity, positive predictive value, negative predictive value] were used to assess the performance of renal functionadjusted D-dimer thresholds versus conventional D-dimer thresholds. Multivariable linear regression was used to
A

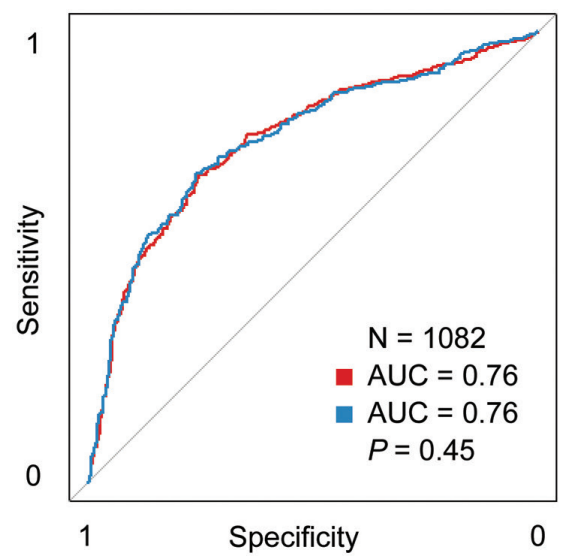

C

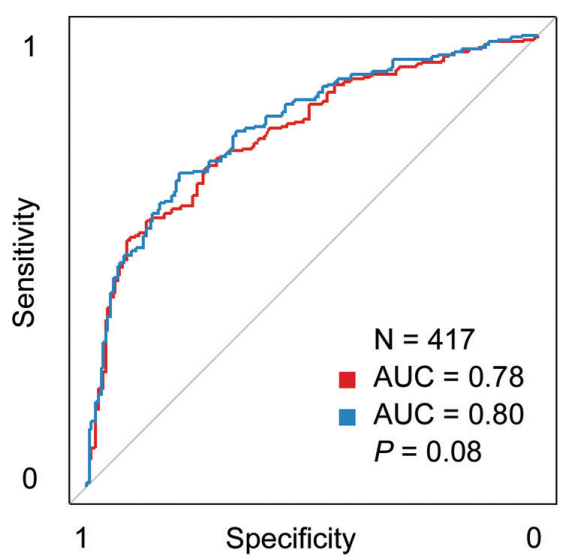

B

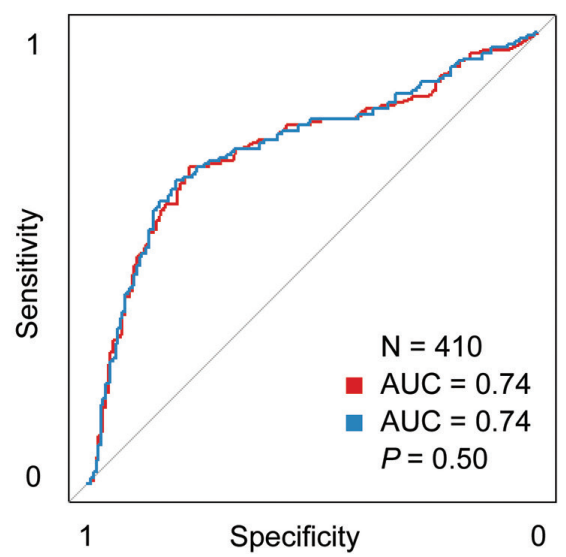

D

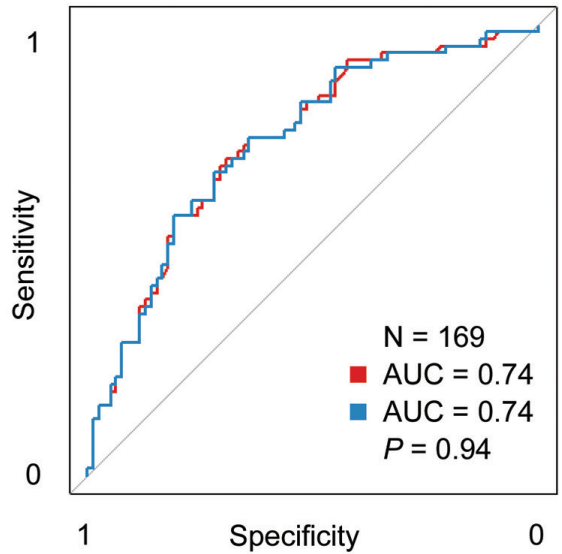

Figure 1. Diagnostic performance of D-dimer versus renal function-adjusted D-dimer, by stage of renal dysfunction. (A-D) These plots depict receiver operating characteristic (ROC) curves for D-dimer (red) and a predictive model combining D-dimer and estimated glomerular filtration rate (eGFR) (blue) in different categories of renal function: total study sample (A), eGFR $\geq 90 \mathrm{~mL} / \mathrm{min} / 1.73 \mathrm{~m}^{2}$ (B) eGFR 60 to $89 \mathrm{~mL} / \mathrm{min} / 1.73 \mathrm{~m}^{2}$ (C) and eGFR <60 mL/min/1.73 m² (D) The limited number of patients with an eGFR $<30 \mathrm{~mL} / \mathrm{min} / 1.73 \mathrm{~m}^{2}(\mathrm{n}=29)$ precluded separate ROC analysis of this subgroup. Hence, this subgroup was merged with the category of patients with an eGFR of $30-59 \mathrm{~mL} / \mathrm{min} / 1.73 \mathrm{~m}^{2}$ for this analysis. $P$ values for differences were calculated using the method of DeLong et al. (see Online Supplementary Text S1 for the reference). 
assess the relationship between renal function and Ddimer levels, accounting for age and CRP concentration. More information on the methodology used is included in Online Supplementary Text S1.

The characteristics of the patients included in the study $(n=1,082)$, stratified by category of renal function, are summarized in Table 1 . The overall mean age was $58.7 \pm 16.8$ years, and the majority of patients were outpatients $(91.4 \%)$ and patients with a low pre-test probability of VTE $(71.9 \%)$. Nearly half of all patients in the study sample were diagnosed with VTE. Overall, the median D-dimer concentration was 1,400 $\mu \mathrm{g} / \mathrm{L}$ (interquartile range: $3,120 \mu \mathrm{g} / \mathrm{L})$. The mean eGFR of patients in this sample was $82.6 \pm 24.3 \mathrm{~mL} / \mathrm{min} / 1.73 \mathrm{~m}^{2}$. The median concentrations of D-dimer and CRP increased monotonically across categories of declining renal function.

Performance metrics of the conventional and ageadjusted D-dimer thresholds (for definitions see Online Supplementary Table S2) according to renal function are displayed in Online Supplementary Table S3. In Online Supplementary Table S4, we included a diagnostic performance comparison in our sample of the three renal function-based D-dimer thresholds that have so far been published. ${ }^{4-6}$ In this sample of patients with variable eGFR values, it is noteworthy that the age-adjusted $\mathrm{D}$-dimer threshold outperformed all other thresholds in terms of positive and negative predictive values.

As shown in Figure 1, we did not find evidence that the inclusion of eGFR significantly improves the D-dimerbased prediction of VTE. The subgroup of patients with eGFR values in the range of 60 to $89 \mathrm{~mL} / \mathrm{min} / 1.73 \mathrm{~m}^{2}$ was the only group in which the difference between AUC had a low random error probability $(P=0.08)$. Nevertheless, the observed difference in AUC $(\triangle \mathrm{AUC}=0.02)$ was so small as to be negligible in terms of clinical benefit, especially since the difference between the two curves was imperceptible in the region of the receiving operating characteristic curve space in which sensitivity exceeds an appropriate threshold for safe exclusion of VTE (e.g. 0.95). All other categories of renal function showed no improvements. Online Supplementary Table S5 reflects that this result was invariant to sensitivity analyses performed across clinically relevant subgroups (e.g. low pretest probability, old vs. young individuals, deep vein thrombosis $v s$. pulmonary embolism), with the exception of outpatients and patients with high pre-test probabilities according to the Wells' score for either deep vein thrombosis or pulmonary embolism. Restricting the sample to outpatients rendered the difference between Ddimer alone and eGFR-adjusted D-dimer in individuals with eGFR values between 60 and $89 \mathrm{~mL} / \mathrm{min} / 1.73 \mathrm{~m}^{2}$ statistically significant, although still not to a clinically relevant extent ( $\triangle$ AUC: $0.02, P=0.049$ ). Among patients in the same renal function category, but with high Wells' pre-test probabilities, the difference was substantive ( $\triangle$ AUC: $0.10, P=0.028$ ); in this category of patients, however, current clinical algorithms advise foregoing $\mathrm{D}$-dimer testing in favor of direct imaging.

To test whether the relationship between eGFR and Ddimer concentrations was affected by age and systemic inflammation, we performed a series of regression analyses (Online Supplementary Table S6). The $\mathrm{R}^{2}$ of the regression predicting $\mathrm{D}$-dimer concentrations with only eGFR indicates that only about $4 \%$ of the variance in $\mathrm{D}$-dimer concentrations was attributable to the eGFR of patients. Including age in the corresponding linear regression model attenuated the partial slope of eGFR by $64 \%$ $(\Delta \beta=0.007)$ (Online Supplementary Table S6) and rendered it insignificant $(P=0.08)$. Including age did not improve the percentage of variance accounted for by the model. A model retaining age, but omitting eGFR, explained the variance in $\mathrm{D}$-dimer marginally better $\left(+8 \% \Delta \mathrm{R}^{2}\right)$.

In Figure 2, we illustrate the influence of age and CRP concentration on the relationship between D-dimer levels and renal function using boxplots, depicting crude and adjusted D-dimer levels against categories of eGFR. By

Table 1. Patients' characteristics by renal function.

\begin{tabular}{|c|c|c|c|c|c|}
\hline \multirow{2}{*}{\multicolumn{2}{|c|}{ Total Sample }} & & \multicolumn{3}{|c|}{ GGFR $\left[\mathrm{mL} / \mathrm{min} / 1.73 \mathrm{~m}^{2}\right]$} \\
\hline & & $\geq 90$ & $60-89$ & $30-59$ & $<30$ \\
\hline Sample size, n. (\%) & $1,082(100)$ & $410(37.9)$ & $417(38.5)$ & $140(12.9)$ & $29(3)$ \\
\hline Age (years), mean (SD) & $58.7(16.8)$ & $45.6(13.6)$ & $65.6(12)$ & $72.2(11)$ & $73.7(11.4)$ \\
\hline Female sex, n. (\%) & $567(52.4)$ & $224(54.6)$ & $217(52)$ & $78(55.7)$ & $14(48)$ \\
\hline \multicolumn{6}{|l|}{ Characteristics related to diagnosis } \\
\hline Diagnosis of VTE, n. (\%) & $515(47.7)$ & $182(44.4)$ & $206(49.5)$ & $64(45.7)$ & $14(48)$ \\
\hline Provoked etiology, n. (\%) & $286(56.3)$ & $111(61.7)$ & $111(54.9)$ & $32(50)$ & $6(43)$ \\
\hline DVT, n. (\%) & $452(41.8)$ & $167(40.7)$ & $181(43.5)$ & $55(39.3)$ & $10(35)$ \\
\hline Proximal DVT, n. (\%) & $254(56.2)$ & $88(52.7)$ & $100(54.9)$ & $34(63)$ & $4(40)$ \\
\hline PE, n. (\%) & $276(25.5)$ & $66(16.1)$ & $116(27.8)$ & $46(32.9)$ & $10(35)$ \\
\hline Isolated PE, n. (\%) & $63(22.8)$ & 15 (22.7) & $25(22)$ & $9(20)$ & $4(40)$ \\
\hline Low-to-moderate PTP, n. (\%) & $597(71.9)$ & $329(80.6)$ & $296(71.3)$ & $87(62.6)$ & $15(52)$ \\
\hline Outpatient, n. (\%) & $989(91.4)$ & $380(92.7)$ & $379(90.9)$ & $125(89.3)$ & $22(76)$ \\
\hline \multicolumn{6}{|l|}{ Humoral biomarkers } \\
\hline D-dimer $(\mu \mathrm{g} / \mathrm{L})$, median $(\mathrm{IQR})$ & $1,400(3,120)$ & $950(1,880)$ & $1,500(3,310)$ & $1,640(3,920)$ & $2,250(3,780)$ \\
\hline $\mathrm{CRP}$ (mg/L), median (IQR) & $8.1(22.4)$ & $5.8(20.3)$ & $8.3(22.1)$ & $8.9(19.7)$ & $39(66.1)$ \\
\hline
\end{tabular}

Categorical variables are presented as relative and absolute frequency, and continuous variables are presented as mean with standard deviation or median with interquartile range (depending on distribution). Frequencies do not incorporate missing information. Stages of chronic kidney disease were classified according to current recommendations of the international guideline group Kidney Disease Improving Global Outcomes (KDIGO). Low-to-moderate pre-test probability refers to a deep vein thrombosis Wells' score of 2 or less or a pulmonary embolism Wells' score of 4 or less. eGFR: estimated glomerular filtration rate; SD: standard deviation;VTE: venous thromboembolism; DVT: deep vein thrombosis, PE: pulmonary embolism; PTP: pre-test probability; IQR: interquartile range; CRP: C-reactive protein. 
A

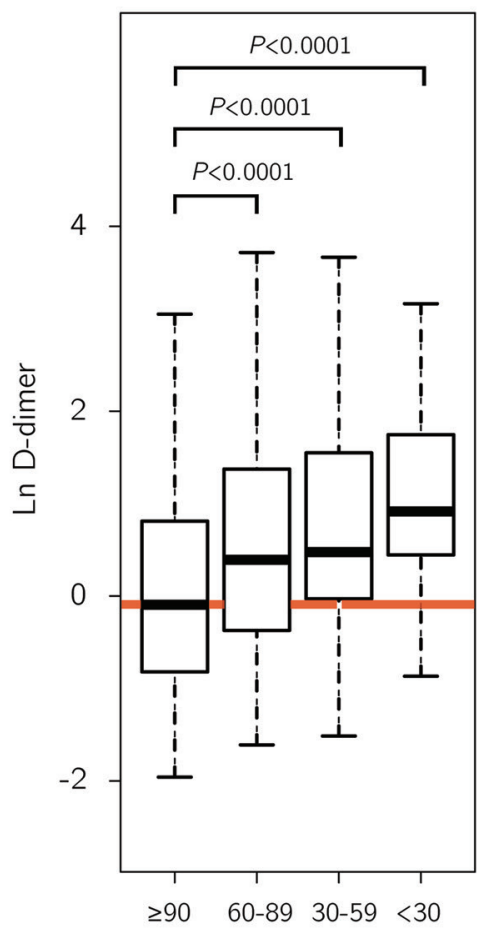

B

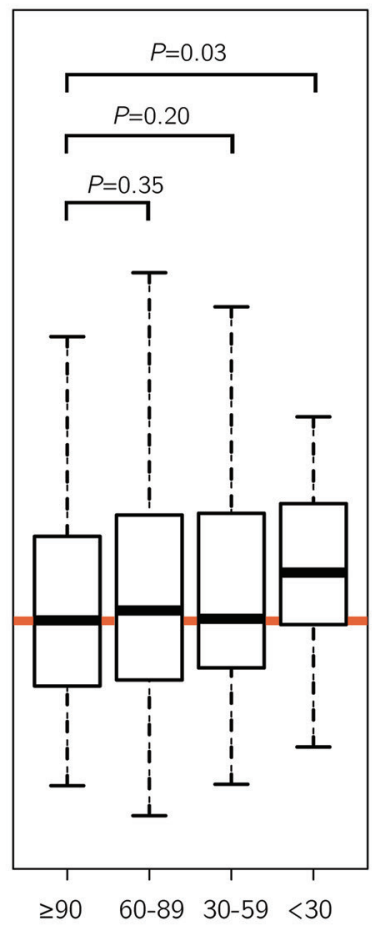

C

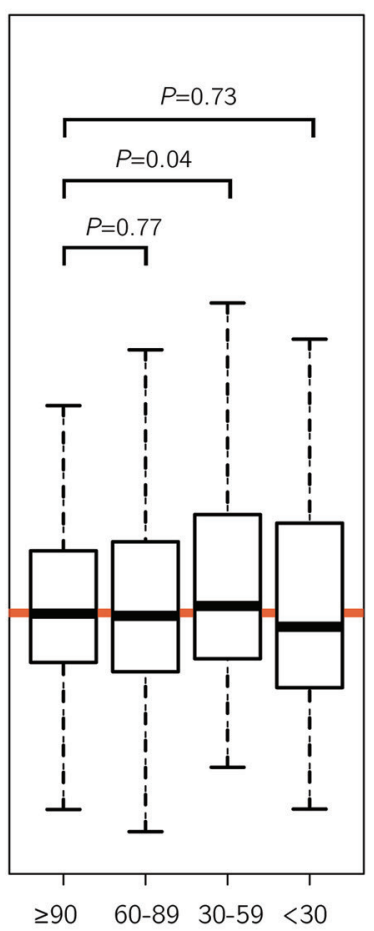

eGFR in $\mathrm{mL} / \mathrm{min} / 1.73 \mathrm{~m}^{2}$

Figure 2. Relationship between D-dimer and estimated glomerular filtration rate after adjusting for age and C-reactive protein levels. (A-C) These boxplots depict the relationship between estimated glomerular filtration rate (eGFR) and D-dimer levels: crude relationship (A) and after adjusting for age (B), and age and C-reactive protein (CRP) levels. (C) The red line indicates the median of the leftmost renal function category ( $\geq 90)$ in each panel. Panels (B) and (C) depict eGFR categories against the residuals of a linear regression model predicting D-dimer concentrations with age and age and CRP, respectively. The $P$ values were derived from Mann-Whitney $\mathrm{U}$ tests.

displaying a slight vertical deviation from the other boxplots, the rightmost boxplot in Figure $2 \mathrm{~B}$ suggests that age did not fully account for the effect of renal function in patients with an eGFR below $30 \mathrm{~mL} / \mathrm{min} / 1.73 \mathrm{~m}^{2}$, i.e. patients with severe renal dysfunction. Figure 2C shows that no visibly discernible trend remains in the $\mathrm{D}$-dimer residuals across categories of renal function after additionally adjusting for CRP levels. While this effect appears to be slightly more pronounced in patients with deep vein thrombosis than in those with pulmonary embolism, the same overall trend is apparent in both (Online Supplementary Figure SF1). In the corresponding linear regression model, age and CRP level were both highly significant $(P<0.001)$ and together accounted for $27 \%$ of the variance in D-dimer (Online Supplementary Table S6), which constituted a marked increase in $\mathrm{R}^{2}$ of the model $(+524.4 \%)$ as compared to the reference model containing only eGFR. Adding eGFR to this model did not improve the variance explained by the model $\left(\Delta \mathrm{R}^{2}=0.004\right)$, and eGFR was not statistically significant $(P=0.11)$ in a model adjusted for age and CRP concentration.

This study is the first to demonstrate that a renal function-adjusted D-dimer test carries no clinically significant benefit in the setting of suspected VTE. Additionally, it is the first study to provide a detailed breakdown of the effect of renal function on D-dimer concentrations. Here, it was shown that eGFR is in large part a proxy for aging: age-adjusting the $\mathrm{D}$-dimer test results all but nullified the effect of renal function on $\mathrm{D}$-dimer concentrations, and a predictive model incorporating age instead of eGFR out- performed the model combining both in terms of total variance explained. When considering separate categories of renal dysfunction, it became apparent that age did not adequately account for the rise in D-dimer concentrations only in individuals with severe renal dysfunction $\left(e G F R<30 \mathrm{~mL} / \mathrm{min} / 1.73 \mathrm{~m}^{2}\right)$. These findings support adjusting the $\mathrm{D}$-dimer test for age, as doing so will likely be sufficient to resolve the perceived issue of renal impairment-induced elevation of $\mathrm{D}$-dimer concentrations in the great majority of patients: in our sample of 1,082 individuals, only $2.7 \%$ had an eGFR below 30 $\mathrm{mL} / \mathrm{min} / 1.73 \mathrm{~m}^{2}$.

In patients with severe renal dysfunction, we found that D-dimer concentrations were substantially elevated, and that CRP level appeared to account for this effect. Associations between coagulation factors such as tissue factor, VIIc, VIIIc, XIIa and fibrinogen and inflammatory markers such as interleukin-6 and CRP have been found in several studies investigating the hypercoagulable nature of severe renal dysfunction, ${ }^{2,8,11}$ and it has been hypothesized that activation of the coagulation cascade in patients with end-stage renal disease is a direct result of the build-up of inflammatory cytokines. ${ }^{1,12}$ Our results align with this hypothesis. An important caveat to this result is that the sample size for this group of patients was limited in the present study $(n=29)$.

The strengths and limitations of this study merit consideration. The present study represents the largest investigation of VTE cases on this topic so far. This study used strict criteria for diagnosis: all study participants were fully examined using color Doppler ultrasonogra- 
phy as well as computed tomographic pulmonary angiography, and board-certified angiologists and radiologists independently adjudicated all diagnoses. By contrast, the majority of the previous studies analyzed historical patients' data, potentially limiting the accuracy of diagnoses. Positive and negative predictive values were based on a higher case prevalence than may be representative of real life; however, these more conservative values were only used for the comparison of thresholds, and for this purpose they were satisfactory. The most notable limitation of the present study is the limited number of participants with severe renal dysfunction in the study sample. The results relating to this group should therefore be interpreted carefully, and not taken to be definitive until they are validated by future studies.

In conclusion, the present study does not indicate any merit from the development of renal function-adjusted D-dimer thresholds for use in individuals suspected of having VTE. In patients with mild to moderate renal impairment, the use of the validated age-adjusted $\mathrm{D}$ dimer threshold should be preferred, whereas in patients with severe renal dysfunction, CRP-adjusted D-dimer testing might be useful.

Vincent ten Cate, ${ }^{1,2,3}$ Markus Nagler, ${ }^{3}$ Marina PanovaNoeva, ${ }^{3,4}$ Lisa Eggebrecht, ${ }^{1,3}$ Natalie Arnold, ${ }^{1}$ Heidrun Lamparter, 1,2 M Iris Hermanns, ${ }^{1,5}$ Hugo ten Cate, ${ }^{6,3}$ Martin H. Prins, ${ }^{2}$ Christine Espinola-Klein, ${ }^{7}$ Thomas Münzel, ${ }^{3,4,7}$ Karl J. Lackner, ${ }^{8,4}$ Philipp S. Wild ${ }^{1,3,4}$ and Jürgen H. Prochaska ${ }^{1,3,4,}$ PSW and JHP contributed equally as last authors.

${ }^{1}$ Preventive Cardiology and Preventive Medicine - Center for Cardiology, University Medical Center of the Johannes Gutenberg University Mainz, Mainz, Germany; 'Department of Epidemiology, Maastricht University Medical Center, MD Maastricht, the Netherlands; ${ }^{3}$ Center for Thrombosis and Haemostasis, University Medical Center of the Johannes Gutenberg University Mainz, Mainz, Germany; ${ }^{4}$ German Center for Cardiovascular Research, Partner Site Rhine-Main, Mainz, Germany; 'Fresenius University of Applied Sciences, School of Chemistry, Biology and Pharmacy, Idstein, Germany; ${ }^{6}$ Thrombosis Expertise Center Maastricht, Cardiovascular Research Institute Maastricht and Maastricht University Medical Center, MD Maastricht, the Netherlands; ${ }^{7}$ Center for Cardiology Cardiology I, University Medical Center of the Johannes Gutenberg University Mainz, Mainz, Germany and Institute of Clinical Chemistry and Laboratory Medicine, University Medical Center of the Johannes Gutenberg University Mainz, Mainz, Germany.

Correspondence: PHILIPP S. WILD -

philipp.wild@unimedizin-mainz.de
JURGEN H. PROCHASKA -

juergen.prochaska@unimedizin-mainz.de

doi:10.3324/haematol.2018.213322

Information on authorship, contributions, and financial \& other disclosures was provided by the authors and is available with the online version of this article at wWw. haematologica.org.

\section{References}

1. Ocak G, Vossen CY, Lijfering WM, et al. Role of hemostatic factors on the risk of venous thrombosis in people with impaired kidney function. Circulation. 2014;129(6):683-691.

2. Sharain K, Hoppensteadt D, Bansal V, Singh A, Fareed J. Progressive increase of inflammatory biomarkers in chronic kidney disease and end-stage renal disease. Clin Appl Thromb Hemost. 2013;19(3):303-308.

3. Robert-Ebadi H, Bertoletti L, Combescure C, et al. Effects of impaired renal function on levels and performance of D-dimer in patients with suspected pulmonary embolism. Thromb Haemost. 2014;112(3):614-620.

4. Lindner G, Funk G-C, Pfortmueller CA, et al. D-dimer to rule out pulmonary embolism in renal insufficiency. Am J Med. 2014;127(4):343-347

5. Xi X, Yang J, Wang Z, Zhu C, Li J, Liu S. Potential utility of a renal function adjusted D-dimer cut-off value for improving the exclusion of pulmonary embolism. Zhonghua Yi Xue Za Zhi. 2015;95(30):2433-2436.

6. Pfortmueller CA, Lindner G, Funk G-C, et al. Role of D-dimer testing in venous thromboembolism with concomitant renal insufficiency in critical care. Intensive Care Med. 2017;43(3):470471.

7. Prochaska JH, Frank B, Nagler M, et al. Age-related diagnostic value of $\mathrm{D}$-dimer testing and the role of inflammation in patients with suspected deep vein thrombosis. Sci Rep. 2017;7(1):4591.

8. Cheung KL, Zakai NA, Callas PW, et al. Mechanisms and mitigating factors for venous thromboembolism in chronic kidney disease: the REGARDS study. J Thromb Haemost. 2018;16(9):17431752

9. Frank B, Ariza L, Lamparter H, et al. Rationale and design of three observational, prospective cohort studies including biobanking to evaluate and improve diagnostics, management strategies and risk stratification in venous thromboembolism: the VTEval Project. BMJ Open. 2015;5(7):e008157.

10. Levey AS, Stevens LA, Schmid CH, et al. A new equation to estimate glomerular filtration rate. Ann Intern Med. 2009;150(9):604612.

11. Nampoory MR, Das KC, Johny KV, et al. Hypercoagulability, a serious problem in patients with ESRD on maintenance hemodialysis, and its correction after kidney transplantation. Am J Kidney Dis. 2003;42(4):797-805.

12. Szotowski B, Antoniak S, Poller W, Schultheiss H-P, Rauch U. Procoagulant soluble tissue factor is released from endothelial cells in response to inflammatory cytokines. Circ Res. 2005;96(12):1233-1239. 